\title{
Innovación, mercados e incertidumbre en la sociología económica clásica
}

\author{
Roberto Herranz González \\ Universidad de Santiago de Compostela \\ roberto.herranz@usc.es
}

Resumen: En este artículo abordamos dos aspectos importantes de la Sociología del Mercado en cuatro grandes clásicos: Max Weber, Emile Durkheim, Georg Simmel y Charles Cortón Cooley. Nos ocupamos del modo en que observaron dos dimensiones relevantes: la innovación y las instituciones. Con relación a la primera observamos el papel que juega la innovación en la división del trabajo y en la expansión de los mercados; y, en cuanto a la segunda analizamos el papel de las instituciones en la construcción de los mercados, en la reducción de la incertidumbre y en el estímulo de la circulación mercantil. El articulo muestra cómo todos ellos adoptan un punto de vista histórico y evolutivo y que, salvo el caso de Weber, eligen una perspectiva opuesta al enfoque estacionario de la economía neo-clásica.

Palabras: sociología económica, mercado, innovación, instituciones económicas, incertidumbre, sociología clásica

Abstract: In this article we address two important dimensions of the sociological approach to markets by such classics as Max Weber, Emile Durkheim, Georg Simmel and Charles Horton Cooley. We observe the way in which they have all analysed two important dimensions: the role of innovation and the role played by institutions. In particular, we look at the role of innovation in the division of labor and the expansion of markets, and analyze the role of institutions in the social construction of markets, the reduction of uncertainty and its performance in the circulation of commodities. The paper shows how they have all adopted an evolutionary and historical perspective and, with the exception of Weber, chosen a dynamic perspective (that is, a critical perspective using the neo-classical approach in economics).

Keywords: Economic Sociology, market, innovation, economy institutions, incertitude and classical Sociology. 


\section{Introducción}

En este artículo se aborda la contribución de cuatro grandes clásicos de la sociología al análisis del mercado. Se trata de figuras como Émile Durkheim, Georg Simmel, Max Weber y Charles Horton Cooley, que escribieron el núcleo de su obra entre 1890 y 1920 y que se aproximaron al estudio sociológico de los mercados desde perspectivas diferentes pero, en buena medida, complementarias.

La vida económica ocupó un lugar importantísimo en sus trabajos. $\mathrm{Al}$ analizarla lo hicieron en clave sociológica, distanciándose de las formas presociológicas dominantes en el ámbito de la economía política clásica y, de un modo más marcado, de la economía neoclásica (Steiner y Vatin, 2009; Swedberg, 1884; Dobbin, 2005).

Todos ellos consideraban que el mercado desempeñaba un papel central para entender la sociedad y el sistema económico capitalista, y observaron de qué modo esta forma de regulación de la economía mantenía una estrecha relación con otras formas como el Estado, la regulación de los derechos de propiedad, las organizaciones, las profesiones y las estructuras comunitarias (Herranz, 2003).

En los siguientes apartados no pretendo contemplar el conjunto de aspectos relativos a la sociología de los mercados que se encierra en su pensamiento. Me limito a descubrir de qué modo se interesaron por el significado de la innovación en las transformaciones económicas y por el papel que juegan las instituciones al bloquear, limitar, encauzar o estimular los comportamientos económicos. Se trata de dos dimensiones estratégicas clave a la hora de observar los cambios en los mercados y el modo en que se reduce la incertidumbre.

Nuestros clásicos interpretaron las estructuras y los procesos económicos como estructuras y procesos sociales, utilizando las herramientas sociológicas que ellos mismos construyeron. Además se inclinaron — como también fue el caso de Simiand, de Veblen y de Schumpeter (Gislain y Steiner, 1995) - por una aproximación a la economía en términos de una estructura social dinámica, en evolución constante, oponiéndose a la perspectiva estática y estacionaria de la economía neoclásica.

\section{La división del trabajo, la expansión de los mercados y la innovación}

En este apartado veremos de qué modo analizaron la creación y la expansión de los mercados, y cómo esta expansión se asocia al desarrollo de la división del trabajo y a los procesos de innovación. 
Comenzaremos conjugando las aportaciones de Durkheim sobre los hechos económicos, la división del trabajo y la innovación, con las contribuciones de Cooley sobre el desarrollo del transporte y sus consecuencias sobre el significado del espacio y el tiempo en los mercados; a continuación, y para finalizar este segundo apartado, nos centraremos brevemente en algunos ensayos de Simmel en los que el autor de la Filosofía del Dinero nos habla del mercado, de la competencia y de la innovación en el contexto de la estructura social y de la cultura urbana (Herranz, 2008). En estas reflexiones Simmel se anticipa, como veremos, a algunos pilares de lo que constituye el núcleo de la teoría de la innovación de Joseph A. Schumpeter.

\subsection{Los hechos económicos como hechos sociales $¥$ un punto de partida para una interpretación sociológica de la economía}

Durkheim sostiene en Las reglas del método sociológico que los hechos económicos son también hechos sociales, es decir, morales, y como tales presentan la propiedad de que son externos a los individuos y tienen un carácter coercitivo, es decir, que los obligan (Durkheim, 1974: 27).

Este es el pilar básico de su proyecto sociológico, sobre el que se construyen las ideas claves con relación al análisis de la economía. Para empezar, el estudio de los hechos sociales que regulan los comportamientos económicos presenta, a diferencia de otros, algunos rasgos singulares. En efecto, en comparación con las creencias religiosas el control social se ejerce de un modo más indirecto y además la moral económica es más laxa. Lo que no significa que carezca de peso pues, como subraya, «tarde o temprano se encuentra un límite imposible de franquear» (Durkheim, 1974: 27).

En cualquier caso, la mayor permisividad y la oportunidad de innovar son dos requisitos para que se mantenga una vida económica fluida: «La moral no puede excederse en la regulación de las relaciones industriales, comerciales, etc., sin paralizarlas y, sin embargo, son vitales [...]» (Durkheim, 1982:278). En realidad, el problema queda indeterminado, lo que permite que se abra un conjunto de interrogantes en relación con el papel de las instituciones y de las corrientes de opinión pública en la configuración de la economía y en la identidad de los actores.

En consecuencia, no es posible que se desarrolle la división del trabajo y que se introduzcan modificaciones dinámicas si las instituciones sociales, y las prácticas de los grupos que las mantienen, son demasiado rígidas. 
A partir de aquí nos interesa subrayar de qué modo evoluciona la división del trabajo y cuál es el papel que desempeña la oferta de nuevos productos, a la par que se desarrollan las necesidades y los mercados se amplían y se vuelven más variados.

\subsection{La densidad dinámica y la evolución de la división del trabajo: el papel de la innovación}

Durkheim considera que las causas que explican el desarrollo de la división del trabajo son múltiples y su dirección imprevisible. Entre otras causas, señala que dicho desarrollo está asociado al volumen, a la concentración de la población y a la mayor comunicación entre diversos grupos sociales, que en las sociedades segmentarias se mantenían separados por barreras culturales y territoriales. Gracias a este contacto, se desarrollan e intensifican los intercambios económicos, aumenta la «densidad material»y, paralelamente, la «densidad dinámica o moral» de la sociedad (Durkheim, 1982:303-306).

De esta manera, el contacto entre las diversas partes, estimulado por la mayor facilidad de comunicación, se acompaña de la creación de normas sociales, de reglas y de valores que facilitan el entendimiento, a la vez que se intensifica la lucha y la competencia por la supervivencia (Durkheim, 1982).

A través de esta lucha, que se desarrolla en mercados más abiertos y más expuestos a la competencia, unas partes desplazan a otras, las empresas compiten entre sí y se producen procesos de subdivisión y especialización, de subordinación o de fusión entre las diversas funciones y unidades productivas, que contribuyen a la configuración de la división del trabajo social. Sin duda, constituye un hecho organizativo de gran alcance.

Los cambios que nos dibuja en La división del trabajo social son un espléndido reflejo de los procesos de concentración industrial-capitalista, que estaban conociendo una intensidad sin precedentes cuando escribía en la última década del siglo xix. El desarrollo de la gran industria y la aparición de la corporación moderna es un fenómeno asociado a lo que se conoce como la Segunda Revolución Industrial.

Estas transformaciones industriales no se producen sin resistencias ni tensiones. Los desgarros sociales que se asocian a la extensión y ampliación de los mercados afectaron brutalmente a las comunidades, a las estructuras familiares y a las identidades de los individuos. La vieja industria y el viejo comercio experimentarán dificultades para adaptarse a los cambios, y finalmente serán incapaces de mantenerse. 
Los recursos liberados por estas crisis se orientan con frecuencia a otras actividades. Pero las adaptaciones y los procesos de movilidad que se requieren son lentos y no se producen de un modo instantáneo. Se trata de fenómenos que, como nos enseña la historia reciente, son recurrentes en la vida económica del capitalismo.

Entre los argumentos que utiliza para dar cuenta de las resistencias a la adaptación de las actividades tradicionales, interesa destacar los fenómenos de lock-in y de bloqueo que sufrió la industria de tradición gremial. En este sentido señala: «Cuando la concentración material y moral del país y la gran industria, que fue su consecuencia, abrieron los espíritus a nuevos deseos, despertaron nuevas necesidades, introdujeron en los gustos y en las modas una movilidad hasta entonces desconocida, la corporación, obstinadamente ligada a sus viejas costumbres, se encontró incapacitada para responder a las nuevas exigencias» (Durkheim, 1982: 30-31).

Además, entre otras causas que favorecen el progreso económico, cabe señalar una condición necesaria para que pueda desarrollarse la división del trabajo. $\mathrm{Si}$ no aparecen nuevos productos, si no se presentan nuevas formas de elaborarlos o si estos procesos no se acompañan de un consumidor más exigente, de nuevas necesidades o nuevas formas de satisfacerlas, dicho desarrollo no puede darse.

En este sentido, Durkheim subraya la importancia de las nuevas condiciones sociales de existencia que se crean con el desarrollo de la división del trabajo, y que conducen a la aparición de nuevas necesidades de consumo y de un consumidor más educado y exigente. Las necesidades, inicialmente latentes, no se cristalizan en demandas si no se presentan nuevos productos que puedan satisfacerlas, es decir, si el sistema productivo y la ciencia no los proporcionan (Durkheim, 1982). El mundo de las necesidades y de los deseos sin límites nos sitúa en el terreno de su preocupación por los estados de anomia y tal vez por los efectos imprevistos de las nuevas sociedades democráticas (Tocqueville, 1978).

Esta explicación de Durkheim parece anticiparse a un interrogante que está presente en los debates más recientes sobre la innovación. Me refiero a la polémica entre la hipótesis technology-push de Schmookler (1966) frente a la hipótesis demand-pull de Mowery y Rosenberg (1979). A diferencia de la una y de la otra, la interpretación evolutiva de Durkheim, sin llegar a formularse con la precisión de las contribuciones más recientes, puede calificarse de «efecto tijera», ya que tiene en cuenta tanto la oferta de nuevos productos como el desarrollo de nuevas necesidades. 


\subsection{El desarrollo del transporte y el nuevo significado econó- mico y social del tiempo y del espacio}

Los factores que Durkheim subraya - que incluyen, entre otros, el desarrollo de las comunicaciones, el volumen y la concentración de la población, así como los espacios que favorecen la innovación y la expansión de nuevas formas de consumo- pueden combinarse y enriquecerse con las aportaciones del resto de autores clásicos. Es el caso de Cooley y su contribución al estudio del transporte.

En su tesis doctoral sobre La teoría del transporte, Cooley sostiene que éste no se puede entender simplemente como un sector económico más, equiparable al resto, ya que desempeña una función fundamental en la organización de la economía y de la sociedad (Cooley, 1969a: 104). Su estudio contempla el papel del transporte en la construcción del mercado nacional en los EE.UU., que, debemos recordar, se convierte en la segunda mitad del xix «en el mercado más amplio y, sobre todo, el que crece con más rapidez en el mundo» (Chandler, 1977: 498).

El desarrollo del transporte y la extensión de los mercados se acompañan de la creación de grandes corporaciones privadas - las nuevas burocracias inicialmente ligadas al ferrocarril- que construyen y gestionan estos servicios. Estas grandes corporaciones, que ocupan una posición estratégica en la sociedad, serán objeto de un estrecho control público.

Cooley era muy consciente de que la ampliación de los mercados se desarrolla sobre la base de una economía organizacional, una economía de grandes corporaciones, que ha dejado su huella en la naturaleza oligopolista de mercados estratégicos y que afecta a la circulación de la información, de la energía y del dinero.

El desarrollo tecnológico del transporte acelera el movimiento de hombres y mercancías, y afecta sobre todo a la comunicación simbólica (de informaciones, de ideas, de conocimientos formalizados), ya que reduce su tradicional dependencia de las servidumbres que impone una menor velocidad del transporte material.

Las mejoras en la eficiencia del transporte cambian profundamente el significado económico y social del tiempo y del espacio. En este sentido nos dice: «El transporte, en razón de su eficiencia, libera a las fuerzas económicas de las cadenas del tiempo y el espacio» (Cooley, 1969a: 88). La revolución del transporte y de las comunicaciones reduce las distancias, acelera el flujo de información, promueve la competencia en mercados más abiertos y extiende las oportunidades de asignación de todos los recursos económicos desde el talento de los directivos hasta la aplicación de los recursos humanos, materiales, financieros y del conocimiento. Además, en la medida en que la comunicación se vuelve más rápida (¡incluso instantánea!) y más barata, la producción se pone fácilmente al alcance 
de numerosos consumidores y la demanda se diversifica, pues sólo cuando los productos son accesibles pueden ser «socialmente deseados» (Cooley, 1966a).

Los fenómenos mencionados, en los que se combinan y refuerzan rasgos de la densidad material y moral, en el sentido de Durkheim, se acompañan de otros efectos no previstos. En La teoría del transporte está en germen la idea de una sociedad del conocimiento que se desarrolla debido a la mayor velocidad de transmisión de la información. En efecto, gracias a la invención y mejora de la imprenta, del telégrafo, del transporte rápido, de la ilustración, «se difunde la información y se hace posible una discusión más rápida y más amplia» (Cooley, 1966: 361-362). Al desarrollo de las fuerzas productivas se incorpora el desarrollo de las fuerzas de la comunicación.

Esta disposición a la novedad se institucionaliza en las economías modernas hasta el punto de que «nuestra vida comercial e industrial ha generado un sistema de comportamientos basado en la perspectiva del cambio» (Cooley, 1956:329). Por este motivo, las sociedades que apoyan la innovación crean universidades que estimulan el «inconformismo» (la figura del innovador de Cooley), respaldan una orientación cosmopolita y contribuyen a la creación de nuevas oportunidades tecnológicas.

La ciencia se presenta como una institución social que facilita la comunicación a escala global. En efecto, y en sus palabras: «A través de sus sociedades científicas, universidades, libros y publicaciones periódicas, bibliografías, etc., mantiene en cierto grado una perfecta comunicación y unidad de esfuerzo entre los científicos a través del mundo» (Cooley, 1969a: 59).

Como el resto de los autores, es plenamente consciente de que en la economía se estaba desarrollando la innovación de base científica. La aplicación industrial de la ciencia, sostenía Weber (1987), contribuye a liberar los productos y los procesos de las ataduras de la tradición. En esta cuestión a Cooley no se le escapa la diferencia entre la invención y la innovación, al señalar cómo los productos de la ciencia que inicialmente carecen de valor de cambio (en el mercado) pueden finalmente ser reconocidos e incorporarse a la circulación mercantil. Las invenciones podrán ser inicialmente reconocidas por una institución profesional, científica o política, y a la larga algunas de ellas podrán valorarse en el mercado de acuerdo con las «reglas» propias de esta institución económica (Cooley, 1966). 


\subsection{La estructura social del medio urbano, los mercados y la innovación en Simmel: algunas afinidades con la teoría de la innovación de Schumpeter}

Los argumentos hasta ahora apuntados mantienen una afinidad con las ideas de Simmel cuando aborda el estudio de la ampliación de los mercados, el crecimiento de la cultura material y el estilo de vida en las grandes ciudades.

Su obra contribuye a enriquecer la explicación sociológica de los mercados en términos dinámicos, una explicación alejada de los modelos estacionarios de la economía neoclásica que tan bien conocía. Además, y como ya señalé, su breve análisis de la innovación, que tal vez ha pasado desapercibido, nos parece pionero y resulta congruente con las aportaciones del resto de autores.

A partir de una interpretación inicial muy próxima a Durkheim, Simmel sostiene que los círculos más pequeños y tradicionales, ligados a un territorio, se ven desbordados por la expansión del comercio y de la economía monetaria. El dinero ocupa aquí un lugar prioritario para entender el desarrollo de la circulación mercantil en círculos más amplios. Precisamente, la ampliación de los mercados y del intercambio entre personas que no se conocen entre sí se asocia a la mayor objetividad y determinación del dinero, así como a la creciente racionalización y objetivación del derecho comercial (Simmel, 1977) ${ }^{1}$.

De esta manera, la sociedad tradicional, formada por círculos concéntricos, se vio desplazada por un tipo de sociedad más abierta, en la que se multiplican los intercambios y los círculos sociales se entrelazan entre sí. En estas nuevas condiciones de existencia, el individuo situado en una red de pertenencias e influencias múltiples está más expuesto a informaciones y a puntos de vista diferentes, y en consecuencia, se presentan oportunidades de individualización y de introducción de combinaciones únicas (Simmel, 1986a).

En su ensayo sobre Las grandes ciudades y la vida del espíritu, escrito en 1903, presenta la ciudad como un espacio que facilita estos procesos de creatividad ( $\mathrm{Si}$ mmel, 1986). En él es posible descubrir «un esbozo de modelo sociológico» sobre la innovación en la vida económica en el que se anticipan algunos de los rasgos centrales de la teoría de la innovación que Schumpeter formulará años más tarde, en 1912.

Para Simmel, la innovación constituye un recurso potencial que permite al empresario, productor o comerciante conquistar al consumidor y protegerse frente a la concurrencia. Las oportunidades de innovación se encuentran en el

1 En este sentido apunta: «al pasar de un círculo estrecho a otro más amplio el derecho se libra de la relación con las meras personas y se convierte en un derecho de las transacciones objetivas. Esta misma evolución es, precisamente, la que caracteriza una realización más detallada de la circulación monetaria» (Simmel, 1977: 425). 
medio urbano, un espacio en el que se concentra la población, se conjuga una elevada densidad material y moral y en donde, bajo el estímulo de la competencia, se desarrolla una intensa actividad creativa. Las grandes ciudades, espacios en los que Simmel descubre la revolución de las cosas, constituyen, actualizando el lenguaje, sistemas urbanos de innovación.

Las grandes ciudades que florecen a finales del siglo XIX se convierten, gracias a una mayor libertad en las relaciones internas y externas, en incubadoras de nuevas ideas. Además, contaban con numerosas ventajas derivadas de su estructura social y de otras economías de aglomeración. En efecto, el elevado volumen de la población facilita la absorción de una oferta más diversificada, a la vez que la agudización de la competencia económica estimula la especialización (Simmel, 1986).

En este sentido precisa: «aquí no sólo fluye la fuente precisamente aludida de la especialización sino la más profundaः el que ofrece debe buscar provocar en el cortejado necesidades siempre nuevas y específicas. La necesidad de especializar la prestación para encontrar una fuente de ganancia todavía no agotada, una función no fácilmente sustituible ${ }^{2}$, exige la diferenciación, refinamiento y enriquecimiento de las necesidades del público» (Simmel, 1986:258)³.

Precisamente en la necesidad de encontrar una fuente de ganancia todavía no agotada reside el núcleo de la idea de Simmel sobre la innovación. Esta exigencia de encontrar un elemento nuevo y diferenciador, nos dice, «obliga al competidor a salir al encuentro del tercero, a satisfacer sus gustos, a ligarse a él, a estudiar sus puntos fuertes y débiles para adaptarse a ellos» (Simmel, 1986a: 304). A lo que añade, algo más adelante, que la competencia «afina en el comerciante la sensibilidad para percibir la inclinación del público y llega a dotarle de una especie de instinto adivinatorio para las mutaciones inminentes de sus gustos, de sus modas, de sus intereses [... $)$ (Simmel, 1986a 305$)$.

En estos fragmentos sociológicos Simmel explica la innovación y la búsqueda de una fuente de ganancia todavía no agotada, considerando los procesos de interacción y de aprendizaje en el mercado y situando este espacio de aprendizaje social en las grandes ciudades. La gran urbe, con una estructura social y económica compleja, se presenta como un espacio muy abierto a combinaciones inéditas y en el que se crean nuevas necesidades (de distinción, de visibilidad, de lucha por el status, etc.) derivadas de las nuevas condiciones de existencia (relaciones impersonales, fugaces, mayores oportunidades económicas y de movilidad social, etc.). El

2 La cursiva es nuestra.

3 En este conocido ensayo sobre la ciudad nos ofrece un buen ejemplo de refinamiento extremo: «Las ciudades son en primer lugar las sedes de la más elevada división del trabajo económica: producen en su marco fenómenos tan extremos como en París la beneficiosa profesión de Quatorzieme: personas, reconocibles por un letrero en sus viviendas, que se preparan a la hora de la comida con las vestimentas adecuadas para ser rápidamente invitadas allí en donde en sociedad se encuentran 13 a la mesa» (Simmel, 1986: 258). 
estudio de la innovación, que constituye sin duda una contribución muy importante de Simmel a la sociología de los mercados, está implícita en diversos fragmentos de su obra y en sus ensayos sobre las formas sociales (Herranz, 2008) ${ }^{4}$.

Como vengo adelantando en este artículo, algunas de estas ideas y fragmentos poseen una estrecha afinidad con la teoría de innovación que Schumpeter comienza a desarrollar de un modo mucho más sistemático ocho años más tarde.

En lo que sigue, deseo subrayar a grandes rasgos los fundamentos sociológicos de la teoría schumpeteriana y apunto a título de hipótesis su proximidad con el interés de Simmel por las formas sociales emergentes.

Uno de los rasgos más sobresalientes de la obra de Schumpeter es el haber propuesto un análisis económico en términos evolutivos introduciendo la Sociología en el núcleo de su argumento. Tal como ha señalado Salvador Giner: «Schumpeter, uno de los más grandes economistas del siglo $\mathrm{xx}$, comparte con Pareto y Veblen la distinción de haber integrado explícitamente la sociología en su propio análisis económico» (Giner, 2004: 401).

Desde los inicios de su carrera académica Joseph A. Schumpeter conocía la obra de Simmel, de Weber y de Durkheim (Swedbeg, 1991) y era muy sensible a las corrientes evolucionistas y a las teorías de la evolución cultural, tal como él mismo reconoce en su Teoría del desenvolvimiento económico ${ }^{5}$, obra en la que se presenta su primer ensayo teórico sobre la innovación.

En su teoría de la innovación adopta una clara perspectiva sociológica al observar cómo el empresario innovador, liderando un proceso que finalmente será imitado por otros, se distancia de las rutinas y de los hábitos de pensamiento que prevalecen en el negocio y en la industria y adopta una nueva combinación, es decir, una nueva función de producción que puede llegar a institucionalizarse.

El interés y la sensibilidad que muestra Schumpeter por los espacios de interacción social que se desarrollan en los diversos momentos por los que atraviesa un proceso de innovación, nos permite establecer un puente con la tradición simmeliana e interaccionista.

\footnotetext{
4 Se presenta, por ejemplo, una cierta analogía entre el tiempo fugaz de (las ganancias derivadas de) las innovaciones que preocupó a Schumpeter y el tiempo transitorio y contingente de la moda Si en el caso de la innovación en los productos la fuente de ganancia es temporalmente contingente, en el caso de la moda el valor del producto como signo de distinción social es también transitorio (Simmel, 1988). Los dos casos, el de la innovación y el de la moda, dan muestra de su éxito económico y social a través de un proceso de imitación (nuevas empresas imitadoras o popularización de la moda) que está en la base de su progresiva devaluación. La moda es también un buen ejemplo de un procedimiento de innovación institucionalizado con importantes consecuencias económicas, en cuyo proceso de construcción actúan numerosos actores económicos.

5 En la primera versión alemana de Teoría del desenvolvimiento económico, que publica en 1912, confiesa que algunas de estas ideas presentadas proceden de 1907 y ninguna es posterior a 1909. Schumpeter observa que la distinción entre estática y dinámica en economía la introduce John Stuart Mill, y éste, indica Schumpeter, «la tomó con toda probabilidad de Comte» (Schumpeter, 1976:15). La utilidad de esta distinción, señala Schumpeter, «ha resultado ser así incluso más allá de las fronteras de la economía, en lo que pudiera denominarse teoría de la evolución cultural» (Schumpeter; 1976: 15).
} 
A ésta y muchas otras contribuciones al estudio, de la innovación a la comprensión de los motivos que guían al empresario innovador y al análisis de sus consecuencias hay que añadir, como indica Ingham, el hecho de que «el proceso mediante el cual el capitalismo se desarrolla con dinero prestado implicaba un alejamiento más del modelo de flujo circular» (Ingham, 2010: 52).

\section{La incertidumbre y las instituciones}

En este tercer apartado presentamos algunos problemas relativos a la regulación conjunta que entraña una economía cada vez más compleja y dinámica, y que está a su vez sometida a numerosas tensiones sociales e intensos conflictos de clase. En otras palabras, nos interesamos por el papel que desempeñan las normas sociales en la determinación de la conducta en los mercados, en la creación de unas expectativas compartidas entre los diferentes actores, y también por el grado en que estas normas son definidas socialmente como legítimas, equitativas o justas.

\subsection{La arquitectura institucional del capitalismo y la determi- nación del comportamiento}

En Economía y sociedad (Weber, 1985) y en Historia económica general (Weber, 1987) nos encontramos con las bases conceptuales y metodológicas de la sociología económica de Max Weber. De la gran diversidad de temas que trata, nos centraremos primero en «la libre mercabilidad» como una de las condiciones «típico ideales» del sistema capitalista, para abordar a continuación algunos de los problemas relativos a la reducción de la incertidumbre en el mercado.

Cuando Max Weber define el capitalismo en términos «típico ideales» presenta una «arquitectura institucional», es decir, un complejo de instituciones que hipotéticamente respaldaría un comportamiento empresarial orientado de acuerdo con una racionalidad económico instrumental, racionalidad que se guiaría exclusivamente sobre la base del cálculo de capital.

A los pilares institucionales básicos del sistema - la propiedad privada de los medios de producción, la inexistencia de trabas a la mercabilidad, la separación de los trabajadores de los medios de producción y la presencia de un mercado de trabajo formalmente libre - añade la calculabilidad plena en el funcionamiento del orden jurídico y administrativo, y la existencia de una ordenación del sistema monetario formalmente racional (Weber, 1985:131). 
La libre mercabilidad es, para Weber y también para Simmel (1977), uno de los pilares del sistema capitalista. Para que se produjese el desarrollo industrial capitalista fue necesario, por una parte, la erosión de numerosas barreras tradicionales y, por otra, un proceso de racionalización sin el cual sería imposible la creación de mercados alejados de las estructuras comunitarias.

Con relación a la primera condición, Geoffrey Ingham señala que para la expansión del capitalismo se necesitó «la erosión de las actitudes tradicionales, la estimulación y satisfacción de nuevas necesidades» (Ingham, 2010: 41). Este tipo de barreras de las que Weber nos da cuenta en numerosos trabajos afectaba a la expansión de los mercados bajo una orientación capitalista, a los modos de trabajar, a las formas de selección laboral y profesional, al significado del dinero, del salario y del beneficio, a la organización de la empresa y del trabajo, así como a las pautas de consumo, de ahorro e inversión.

Algunas de esas estructuras tradicionales convivían con mercados locales, y a través de usos y costumbres, de instituciones locales o de principios arraigados en la conciencia de las gentes, desempeñaban un papel importante controlando a los participantes, sus productos y sus acuerdos, y velando también por su cumplimiento. Pero, de un modo u otro, constituían barreras a la libre mercabilidad, ya que imponían limitaciones al cálculo de capital y a los procesos de acumulación.

En cuanto a la segunda condición, es decir al proceso de racionalización, constituye, como apuntó Simmel, una condición para el desarrollo del capitalismo y su ausencia podría considerarse como otra forma de limitación de las oportunidades de ampliación de los mercados y del desarrollo de la mercabilidad.

Numerosas instituciones, como el dinero, el derecho comercial o la organización social del tiempo, ayudan a explicar la determinación de los comportamientos y el desarrollo de los mercados (Simmel, 1977). En efecto, la precisión, la puntualidad, la estandarización, la formalización de tareas y de procedimientos, la justicia formal, penetran en las relaciones económicas facilitando el crecimiento de la circulación mercantil y la expansión de los mercados.

Todas ellas promueven la determinación de la conducta, la comparación y el cálculo, y por consiguiente facilitan los intercambios y contribuyen a eliminar numerosas fuentes de incertidumbre. Parece claro que su eficacia será mayor en la medida en que se incorporen a la cultura y el lenguaje de sociedad, a su sistema de significados y a lo que se da por sentado.

En este punto cabe adoptar una sabia cautela, observando, como nos enseña la sociología clásica, algunas posibles consecuencias latentes de la racionalización, tales como la aparición de nuevas formas de incertidumbre, la rigidez de la formalización o los problemas de legitimidad del orden social. 
En efecto, al eliminar algunas fuentes de indeterminación y facilitar la extensión de los mercados se crean nuevas condiciones económico-mercantiles, una división del trabajo más compleja y una estructura de poder que generan y promueven nuevas fuentes de incertidumbre (la mayor velocidad de los intercambios, la renovación de productos y servicios, la complejidad de los compromisos, la competencia, la naturaleza volátil del dinero y del capital, etc.).

Por otra parte, no hay que olvidar que estos procesos de racionalización pueden acarrear consecuencias no esperadas que contribuyan a bloquear los procesos de adaptación e innovación en la vida económica y en los mercados. Los efectos latentes de la formalización que tuvieron una resonancia en la sociología de las organizaciones a mediados del siglo xx ya preocupaban a Durkheim, a Simmel y a Cooley unas décadas antes.

Como advertía Durkheim (1982), en la vida económica son necesarias las reglas, pero éstas deben ser lo suficientemente flexibles para que permitan la innovación y los procesos de adaptación. En un sentido más centrado en las empresas y organizaciones, Simmel (1986a) pensaba que las formas burocráticas que se aplican a las grandes organizaciones limitan la «variabilidad» y la desviación de los comportamientos, y en consecuencia la innovación. Finalmente, el formalismo excesivo, y «el ritualismo» que puede derivarse de él, sería adecuado para los procesos de producción en masa en la industria, pero impiden los comportamientos innovadores, la comprensión del conjunto del proceso productivo y la adaptación a los cambios (Cooley, 1966).

Algunas ideas de Cooley parecen anticiparse a la perspectiva contingente (Burns y Stalker, 1961) de la sociología de las organizaciones, que nos permite dar cuenta de las estructuras y espacios económico-productivos más innovadores y orientadas a mercados dinámicos (Herranz, 2007).

Finalmente, la falta de legitimidad de las instituciones en las que reposa el mercado es también una fuente de incertidumbre. La legitimidad de estas instituciones, ya sea de la propiedad y sus nuevas formas, del (uso del) dinero o de la mercabilidad, es, en realidad, una variable más que un dato. Como nos enseña Weber (1985), la creencia en la legitimidad de los pactos y de los órdenes sociales estatuidos en el ámbito económico refuerza la estabilidad de las relaciones. Por el contrario, la falta de legitimidad significa una disociación entre las normas oficiales hegemónicas y dominantes y las definiciones que de ellas hacen los individuos y los grupos sociales.

Tras interesarse por las condiciones que favorecen la mercabilidad, Weber hizo un importante esfuerzo por analizar el mercado en términos de un espacio de participación social, manteniendo una tensión analítica entre las construccio- 
nes típico ideales del capitalismo y de los mercados y la variedad en términos tanto históricos como societales.

Entiende el mercado como un espacio de interacción social con motivo del intercambio y bajo condiciones de competencia y de regateo. El análisis de cada uno de estos aspectos, de los actores que participan, de las capacidades y de las orientaciones que llevan consigo, de los productos que se intercambian, de los medios utilizados para llegar a un acuerdo y de los resultados constituye una base de partida en el desarrollo de una sociología de los mercados (Clark, 1991) ${ }^{6}$.

Una vez que se llega a un acuerdo en el mercado, dice Weber (1985), es necesario cumplirlo y, además, hacerlo de buena fe. Aquí reside otra de las fuentes de incertidumbre del mercado asociada a los costes de transacción.

Entre los motivos que favorecen el cumplimiento de los acuerdos de mercado destaca el interés a la hora de mantener una relación a lo largo del tiempo, la ética de mercado, la reputación y la desaprobación social ${ }^{7}$, y finalmente el control de la comunidad y de las asociaciones intermedias, como las profesiones o los consejos reguladores.

Todos estos factores pueden contribuir a garantizar el cumplimiento de los acuerdos de mercado, si bien en su opinión, y alejándose del papel que les atribuiría Durkheim, presentan numerosas debilidades en el contexto del capitalismo moderno. Para Weber, en un contexto social sin precedentes en cuanto a la agudeza de los antagonismos, es el Estado, a través del Derecho, quien desempeña este papel de salvaguarda y el que se encargaría de establecer las condiciones marco para el desarrollo del intercambio económico: «El poderío universal de la sociedad que constituye el mercado demanda [...] un funcionamiento del derecho calculable según reglas racionales» (Weber, 1985: 272).

\subsection{La organización social de los mercados en Durkheim}

Precisamente la piedra angular de La división del trabajo social consiste en un análisis dinámico de las relaciones, unas veces en tensión y otras en armonía, entre la estructura social de la economía y el sustrato institucional. No podría desarrollarse una economía ni tampoco podrían existir unas relaciones fluidas en el

\footnotetext{
6 El estudio del mercado interesó de un modo muy especial a Weber, aunque la atención que se le prestó durante años ha sido reducida. En particular interesa el capítulo 2, que versa sobre «Las categorías sociológicas de la vida económica», y de un modo muy especial el Capítulo VI de la 2a parte, titulado «Mercado (incompleto)», así como otras contribuciones menores. Si Marx dejó incompleto en El capital el capítulo de clases sociales, Weber nos legó también incompletos unos apuntes sobre las clases sociales y sobre el mercado. El Capítulo VI de Economía y sociedad se titula «Mercado (incompleto)».
}

7 Esto sucede incluso en los mercados aparentemente más impersonales, como los mercados financieros (Weber, 1987). 
mercado si no existiesen unas relaciones institucionalizadas y unas expectativas sociales compartidas.

La posición de Durkheim es intelectualmente hostil a la de los economistas liberales al considerar que el actor económico es un ser moral, lo que significa que ha sido socializado y se encuentra él mismo ante una estructura social, con las limitaciones y los recursos de una herencia social. El proceso de individualización que estudia y al que contribuye la misma sociedad no devuelve al individuo a un estado natural.

Además, los economistas liberales no eran conscientes de que, de un modo paralelo a las relaciones contractuales de los intercambios económicos, se desarrollan a su vez compromisos que no tienen un carácter contractual, que son externos a las intenciones y a las decisiones de las partes, es decir, a los acuerdos propiamente dichos (Durkheim, 1982: 429). Estas relaciones, que son externas a la interacción de las partes, contribuyen a cimentar los intercambios: unas veces se basan en el propio contexto legal, del que no tienen que ser necesariamente conscientes las partes, mientras que en otras se fundan en la costumbre y en las reglas de conducta sancionadas socialmente (Macaulay, 1963).

El valor atribuido a las normas sociales no contractuales está claro. Mientras que para los utilitaristas todo tipo de regulación (moral) o vínculo no contractual sería un elemento residual, ajeno al mercado, limitativo de su acción, para Durkheim tales regulaciones constituirían un factor necesario para su desarrollo y, además, tendían a adquirir más importancia, a pesar de los espacios de indeterminación, a medida que la división del trabajo y la especialización por industrias y profesiones se volviese más compleja y diferenciada. Estas normas extrañas al contrato propiamente dicho permiten reducir la incertidumbre y las limitaciones del contrato.

En efecto, la definición de las condiciones de cooperación en el intercambio plantea un problema de conocimiento, previsión e información que afecta a cualquier tipo de contrato. Si en el desarrollo de los intercambios hubiese que negociar en cada momento y en cada instante las condiciones precisas, si hubiese que especificar todo tipo de contingencia por adelantado, los intercambios se paralizarían (Durkheim, 1982). En relación con este punto, señala: «es preciso también que la forma como deben concurrir, si no en toda clase de encuentros, al menos en las circunstancias más frecuentes, sea predeterminada. De otra manera serían necesarias en cada instante nuevas luchas para que pudieran equilibrarse [...]. Pero no es ni necesario, ni incluso posible, que la vida social se deslice sin luchas. El papel de la solidaridad no es suprimir la concurrencia, sino moderarla» (Durkheim, 1982: 429-430). 
De este argumento puede deducirse que si la sociedad (sociedades) hereda (heredan) conocimientos y formas de cooperación y de intercambio en las que se socializan los individuos sirviéndoles de referencia en su comportamiento, también sucede que a medida que se desarrolla la división del trabajo, y para que ésta se adapte a las nuevas circunstancias, se crea un complejo mundo de normas en torno al modo en que se desempeñan los roles y funciones, se definen los productos, se establecen los intercambios y se resuelven los conflictos.

Al mismo tiempo Durkheim, en un punto de tensión con lo dicho hasta el momento, es muy consciente de cómo el desarrollo de la división del trabajo y la expansión de los mercados se acompaña por un lado de vacíos normativos y por otro de la presencia de situaciones, de normas o de acuerdos que se consideran injustos.

En relación con el primer asunto, Durkheim aprecia un déficit de regulación jurídica y moral en diversos ámbitos de la vida económica. Mientras que, en su opinión, existe una moral profesional en el mundo jurídico, educativo, sanitario y religioso, en cambio no están claras ni bien definidas en «las relaciones del patrono con el empleado, del obrero con el jefe de empresa, de los industriales en competencia unos con otros o con el público» (Durkheim, 1982:2).

En cuanto al segundo asunto, pensaba que la propuesta de nuevas normas puede erigirse en una respuesta de la sociedad frente a situaciones socialmente definidas como abusivas o fruto de acuerdos que hieren la conciencia pública y que serían una expresión de la desigualdad de fuerzas en el mercado. De ahí que abogase por la institucionalización de las relaciones obrero-patronales, la creación de un sistema de protección social y la reforma del sistema de herencia que impedía la igualdad de partida en la competencia en el mercado (Durkheim, 1982).

No se trataba tan sólo de cubrir un vacío normativo, que regulase los comportamientos y contuviese las expectativas, sino de que dichas reglas se considerasen socialmente como justas. Las representaciones sociales en torno a la justicia forman parte de las preocupaciones de Durkheim con relación a la organización social de los mercados, y sigue constituyendo hoy un aspecto relevante en el estudio de las instituciones económicas y de sus resultados.

\subsection{Charles Horton Cooley: la necesidad de la regulación y el problema de la selección adversa}

El análisis de Cooley mantiene una cierta afinidad con Durkheim y con la posición de Karl Polanyi (1989) en La gran transformación, al considerar que la sociedad debería organizar socialmente el mercado, promover estándares que 
permitiesen definir las características de los productos, clasificarlos y establecer los criterios de medida.

A principios del siglo $\mathrm{xx}$, Cooley denunció una atmósfera dominada por un excesivo comercialismo, entendido como la búsqueda de la ganancia sin reparar en los medios, a la vez que reconocía que «los consumidores, sobre los que el principio comercial descarga toda la responsabilidad, carecen de estándares claros y de medios organizados para aplicar aquellos que tienen» (Cooley, 1966: 342-343). Bajo determinadas condiciones en las que la competencia carece de reglas, ya sea porque se oriente exclusivamente bajo el principio de la maximización de beneficios o bien porque exista un vacío de control social y se generalicen prácticas fraudulentas, gana el que tiene mayor habilidad para engañar y, en consecuencia, el producto malo desplaza al bueno y la calidad se resiente. Este es uno de los temas relativos a lo que hoy se denomina selección adversa, fenómeno al que se refiere en sus trabajos (Cooley, 1899).

El sociólogo del self-looking glass sostiene que tan sólo a través de la interacción entre productores y consumidores, de un amplio debate público y de la intervención de criterios fundados en la racionalidad técnica, se irían desarrollando nuevos estándares y sistemas de medida orientados a mejorar la calidad y la información en los mercados.

Para encontrar una solución a una situación que juzgaba como muy preocupante, proponía un control de la sociedad sobre el mercado mediante un proceso de estandarización que permitiese definir los comportamientos legítimos y clasificar las competencias, las capacidades y los productos. Se trataba de establecer sistemas de medida que facilitasen las comparaciones y las transacciones. Los estándares de cualquier tipo, siempre que se acepten socialmente, permiten establecer relaciones claras, «facilitan la cooperación y mitigan o alivian los conflictos» (Cooley, 1966: 385).

Este proceso de normalización puede afectar igualmente a la organización de la empresa y del trabajo mediante la estandarización de los procedimientos y de los métodos de evaluación del trabajo.

La presencia de normas estandarizadas contribuiría a reducir la desconfianza y a aligerar las transacciones, mientras que su ausencia resulta una fuente de insatisfacción que afecta al desarrollo de la vida económica. La existencia de estándares permite, por añadidura, construir la información relevante con relación a las mercancías mediante su categorización, clasificación y graduación. Es, por tanto, una dimensión crucial para organizar cualquier mercado.

No me gustaría terminar esta reflexión sobre Cooley sin dar cuenta del modo en que denuncia prácticas económicas injustas de las que se derivaban, además, 
importantes costes sociales. En su opinión, es la inteligencia de la sociedad la que debe impedir la explotación de los niños, aspecto que limita el desarrollo futuro de los recursos humanos o el deterioro medioambiental (Cooley, 1966). Este es un asunto que nos sugiere también la importancia que en la sociología económica debe atribuirse a las externalidades negativas y al modo en que se definen socialmente como costes.

Sin lugar a dudas, los problemas relativos a los límites sociales de la mercabilidad interesaron de modos diversos a todos los clásicos de la sociología económica, lo que nos ayuda a observar y comprender de qué forma se construyen socialmente los mercados y cuáles son los ámbitos en donde la sociedad no considera legítima la lógica mercantil.

\subsection{La confianza, el secreto y la mentira en las estructuras y los procesos económicos: la contribución de Simmel}

La sociología del mercado se interesa por el modo en que se construye socialmente la información, por las asimetrías informativas ligadas a la estructura social y por el papel que desempeña la confianza, el secreto, la ocultación y la mentira en la vida económica.

Las aportaciones de Simmel pueden considerarse pioneras, pues nos permiten caracterizar, como él mismo nos sugiere una y otra vez, la estructura social de la economía en términos de «distancias sociales», de proximidad y de lejanía. La economía puede contemplarse como una especie de geometría variable en la que las distancias entre los actores cambian en razón del grado de confianza, de la apertura o del secreto, de la información veraz o de la mentira que se alberga en el entramado de las relaciones sociales. y, claro está, en función del dinero.

El grado de confianza que podemos depositar en los otros es una característica en cualquier tipo de relación social, incluidas las económicas. La confianza, nos dice Simmel, se sitúa a medio camino entre el conocimiento absoluto y la ignorancia: «El que sabe no necesita confiar; el que ignora no puede siquiera confiar» (Simmel, 1986a: 367). En efecto, la confianza se basa en algún tipo de indicio que reduce la incertidumbre con respecto al modo en que esperamos que se comporten los otros.

La necesidad de confiar en los demás actores, en sus capacidades y en las instituciones constituye una condición necesaria para el desarrollo de una economía compleja, innovadora y dinámica. Cuando la confianza en los mercados financieros se resiente, la economía se paraliza. La confianza es fundamental en una economía que es cada vez más una «economía de crédito», y en este sentido Simmel 
apunta que «mucho más ampliamente de lo que suele pensarse descansa nuestra existencia moderna sobre la creencia en la honradez de los demás, desde la economía, que es más economía de crédito, al cultivo de la ciencia, en la cual los investigadores, en su mayoría, tienen que aplicar resultados hallados por otros y que ellos no pueden comprobar. Construimos nuestras más transcendentales resoluciones sobre un complicado sistema de representaciones sociales, la mayoría de las cuales supone la confianza de que no somos engañados» (Simmel, 1986:363).

El tejido social de la economía, con sus lazos y comunicaciones, está igualmente ligado al secreto. En sus reflexiones nos recuerda que el secreto da un tono tanto a las relaciones más íntimas como a las relaciones dentro de un grupo y entre grupos. El secreto como forma social puede ser legítimo o ilegítimo, puede derivar de un compromiso individual o de una exigencia institucional.

Son muchos los ejemplos que podemos extraer de la vida económica. El secreto bancario puede ser legítimo aunque en ocasiones encubre y favorece prácticas al margen de la ley. Las empresas más innovadoras - y sus departamentos de $\mathrm{I}+\mathrm{D}+\mathrm{i}$ - tratan de proteger celosamente el secreto industrial frente a la competencia. También, y como sucede con el conocimiento tácito, resulta más fácil mantener a salvo un procedimiento innovador dada la dificultad de transmisión codificada y al margen de una relación de proximidad.

Mas el secreto, como sucede en el ámbito de la economía sumergida e irregular, en los espacios de corrupción y en la economía criminal, es un medio para ocultar actividades ilegítimas.

Hay formas de secreto que afectan a las instituciones económico-formales y que introducen prácticas informales socialmente inadmisibles. De ahí los esfuerzos (en las democracias) por introducir transparencia en los más variados órdenes institucionales. En la Filosofía del dinero, Simmel presenta un tema de gran interés para la sociología económica, relativo a la transparencia de las cuentas públicas y de la Administración en las grandes sociedades anónimas ${ }^{8}$. Esta observación, recogida en la nota a pie de página, pone de relieve que la falta de transparencia y los problemas de información y de manipulación de la información que están en la base de la crisis económica internacional de 2008 no son temas nuevos sino que vienen repitiéndose, ahora a escala más ampliada, a medida que se desarrolla el capitalismo y se globalizan los mercados.

Además de la ocultación y el secreto, la mentira supone una información manipulada. La mentira tiene tal grado de importancia que «las estructuras socio-

8 «Si los órdenes jurídicos modernos imponen el carácter público de la contabilidad de los Estados y de las sociedades anónimas, en realidad los peligros que se tratan de evitar emanan, principalmente, de la forma monetaria de las actividades, de la facilidad de la ocultación, del cálculo erróneo, del empleo ilegítimo, todos los cuales constituyen peligros para los terceros que [...] únicamente se pueden evitar, en cierto modo, por medio de la publicidad de los negocios» (Simmel, G., 1977: 479). 
lógicas se distinguen de un modo característico según el proceso de mentira que alientan en ellas» (Simmel, 1986: 362).

La manipulación del conocimiento es un medio de poder y una táctica en un juego estratégico que está al alcance de los grupos económicamente dominantes y que mediatiza nuestra interpretación del mundo económico. Como he indicado en otro lugar refiriéndome a los mercados financieros, la racionalidad limitada es cada vez más una racionalidad «mediatizada por expertos» encargados de «diagnosticar los productos financieros y de aconsejar al público» (Herranz, 2007: 186). En realidad, el problema de la incertidumbre en los mercados y la vida económica no puede desligarse de la concentración de la riqueza y del poder.

Por este motivo deseo concluir este trabajo con algunas de las reflexiones de Simmel sobre lo que significa el dinero, reflexiones en las que nos habla del inmenso poder del capital y de los problemas de incertidumbre que se derivan de su volatilidad.

El dinero es, en efecto, una institución social que agiliza las transacciones y que, a diferencia del pago en especie, permite que su propietario decida con libertad en qué gastarlo, en qué momento y en qué lugar, motivo por el cual la libertad de elección es un beneficio, una nueva regalía entre muchas otras, que ha de añadirse al valor del dinero (Simmel, 1977).

Esto también significa que sólo los más ricos, y en mayor medida cuanto más dinero tengan, pueden liberarse de las necesidades más elementales que, como le sucede al pobre, comprometen de antemano buena parte de los ingresos. En este sentido, y refiriéndose al pobre, dice Simmel: «como quiera que su ingreso está predeterminado de antemano, ya que sólo alcanza para las necesidades más elementales de la vida, únicamente permite un escasísimo margen para la elección entre las diversas posibilidades de aplicación» (Simmel, 1977: 248).

Precisamente por esta razón cuanto mayor sea la concentración de la riqueza mayor es la incertidumbre a la que puede verse sometida la sociedad. Estas ideas ponen de relieve el contraste entre el poder de la denominada economía real, que es comparativamente más inelástica y más ligada a la vida, y el poder de la economía financiera.

De esta manera, el sociólogo de Filosofía del dinero muestra un profundo conocimiento del sistema capitalista y del papel del poder, así como de las distancias en la estructura social de la economía. El dinero es un medio para medir estas distancias y un instrumento para ampliarlas o reducirlas.

No carece de excelentes ejemplos que nos revelan lo que ocurría hace más de un siglo, anticipando lo que sucede hoy y poniendo de relieve el modus operandi del capitalismo. El poder de los grupos financieros se manifiesta en la suerte que 
corren en las situaciones de crisis económica. En este sentido apunta Simmel: «La ventaja que el dinero extrae de su libertad frente a los contenidos y movimientos específicos de la economía se manifiesta también en otro tipo de fenómeno, cuyo carácter común es que, incluso en mitad de las sacudidas más fuertes y más ruinosas de la economía, la gente de dinero propiamente dicha acostumbra a obtener los beneficios habituales e incluso a aumentarlos» (Simmel, 1977: 244).

Nos queda por abordar la desconfianza que puede producir la excesiva concentración del poder económico-financiero, que con frecuencia actúa al margen o en contra del control democrático y que dispone de medios para controlar el entorno político y cultural. Pero esto es tema para otro trabajo.

Tal como observamos en relación con las consecuencias latentes de la racionalización, este proceso que conduce a la determinación de numerosos ámbitos del comportamiento crea unas nuevas condiciones de existencia para la economía y la sociedad que, de un modo creciente en un contexto global, generan nuevas fuentes de incertidumbres.

\section{Conclusión}

A pesar de la singularidad de cada uno de los autores al analizar la economía y la sociedad, se encuentran en ellos numerosas similitudes y aportaciones complementarias.

Todos ellos adoptaron una perspectiva histórica y, de un modo que está menos claro en la obra magistral de Weber, abordaron el estudio de la economía en términos dinámicos y evolutivos, incorporando un punto de vista muy distante de la perspectiva estática y estacionaria de los economistas clásicos y de la teoría económica neoclásica.

A lo largo del texto hemos hecho hincapié, en primer lugar, en las causas que explican la expansión de los mercados en su relación con el progreso de la división del trabajo y los procesos de innovación; y en segundo lugar, en el papel de las instituciones y su relación con la reducción de la incertidumbre, la creación de unas expectativas socialmente compartidas y la determinación de la conducta.

Con relación a las causas que permiten la creación y la extensión de los mercados, hemos de subrayar en esta conclusión de qué modo Durkheim analiza la ruptura de los límites a la mercabilidad, uno de los pilares básicos para el desarrollo de la división del trabajo, y que preocupó igualmente a Weber en su estudio del capitalismo.

El contacto entre grupos y territorios previamente aislados, el debilitamiento de las estructuras comunitarias y la revolución de las tecnologías del transporte 
material y de la comunicación «simbólica» (Cooley) crean nuevas oportunidades de intercambio comercial, transformándose así el significado económico y social del tiempo y del espacio.

El crecimiento de las relaciones comerciales se acompaña de una agudización de la competencia, resultado de la cual se produce un intenso proceso de concentración y de especialización industrial. Las nuevas condiciones de existencia estimulan el desarrollo de nuevas necesidades inicialmente latentes, que se cristalizan y determinan en la medida en que el sistema productivo pueda responder a ellas, es decir, en la medida en que pueda innovar. Para Durkheim, sin innovación no es posible el progreso de la división del trabajo.

Este argumento, trazado con los mimbres de Durkheim y de Cooley, es compatible con el escenario que le sirve de referencia a Simmel para explicar la crisis de la sociedad tradicional, el desarrollo de los mercados en círculos más amplios y las condiciones que favorecen los procesos de innovación.

La aportación de Simmel añade valor a la perspectiva evolutiva y estructural de Durkheim y Cooley. En esta conclusión, deseo destacar su visión de las formas de interacción social que caracterizaba a los mercados de las grandes ciudades, espacios que presentaban condiciones sociales muy favorables para el desarrollo de la innovación. Esta interpretación de Simmel concluye magistralmente con una breve observación, cuando afirma que las empresas compiten entre sí «para buscar en el cortejado necesidades siempre nuevas y específicas. La necesidad de especializar la prestación para encontrar una fuente de ganancia todavía no agotada, una fuente no fácilmente sustituible, exige la diferenciación, refinamiento y enriquecimiento de las necesidades del público» (Simmel, 1986:258).

Esta manera de entender la innovación, en la que se descubre parte del argumento que años más tarde utilizará Schumpeter, tiene el valor de enmarcarse en una concepción del mercado entendido como un espacio de aprendizaje a través de la interacción social. Una concepción que nos acerca a interaccionistas como Cooley, quien, del mismo modo que Simmel, no pierde de vista el escenario estructural e institucional más amplio que sirve de referencia a los actores.

En cuanto al papel de las instituciones en la reducción de la incertidumbre, podemos concluir que no hay mercados sin reglas, pues todos los mercados están organizados socialmente, aunque dichas reglas sean impuestas de un modo unilateral o incluso aunque dicha organización no sea considerada legítima por algunos de los actores en juego. El juego contra las reglas es una de las posibles formas de transformación de los mercados.

Weber, y el resto de autores, al interesarse por la industrialización capitalista descubren que su desarrollo está estrechamente ligado a la desaparición de las 
trabas a la mercabilidad y de los lastres que imponían las orientaciones tradicionales y las viejas estructuras comunitarias. Esta es una condición necesaria pero no suficiente. En efecto, es indispensable que a su vez se desarrollen nuevas instituciones que contribuyan a la determinación del comportamiento y a la reducción de la incertidumbre.

En este sentido, Simmel y Weber subrayan los procesos de racionalización que se manifiestan en la creación del dinero, en la organización del derecho racional o en otros fenómenos institucionales, como la organización del tiempo o los procesos de formalización de las actividades y del conocimiento; mientras, Durkheim apunta el papel que desempeñan las expectativas sociales compartidas en la organización del mercado y en el desarrollo fluido de los intercambios.

Durkheim y Cooley también observan la presencia de vacíos normativos, ante lo cual consideran necesaria la creación de reglas negociadas por las asociaciones intermedias. En el caso de Cooley se necesitaría el desarrollo de estándares con relación a los productos y las cualificaciones, de modo que se pudiesen establecer comparaciones, lo que reduciría los conflictos y facilitaría la cooperación y el intercambio.

Esta regulación permite definir la información relevante, reducir la indeterminación y la incertidumbre e introducir una mayor confianza en las relaciones de intercambio y en los compromisos que se adquieren en la vida económica.

Simmel se acercó al problema de la incertidumbre, y al problema derivado de los límites de nuestro conocimiento y de nuestra información, observando algunos de los factores que, como la confianza, la ocultación, el secreto o la mentira, inciden en las «distancias sociales» de la economía, dando un tono concreto a sus estructuras sociales y a la naturaleza de las relaciones de poder.

Finalmente, todos los autores sin excepción son conscientes del problema de la legitimidad. Más allá del establecimiento de normas que agilicen la actividad productiva y de mecanismos que garanticen su cumplimiento, se necesita que dichas normas sean consideradas como aceptables y justas. De este modo se cuestionan, con tonos analíticos o de denuncia, el grado de legitimidad de las formas de mercado y de sus resultados.

Estos asuntos afectan igualmente a las formas de mercabilidad que se consideran tolerables y legítimas, es decir, a los actores que pueden participar en los mercados, a los productos que se pueden producir e intercambiar $y$ a las formas que adoptan los acuerdos. Unos temas que hoy siguen siendo motivo de importantes movimientos sociales, se asocian a diversas corrientes de opinión y generan grandes debates públicos. 
El análisis que se ha presentado sobre la sociología de los mercados en los autores clásicos no pretende ser exhaustivo, ni agota los aspectos que interesan a la sociología económica. A través de ellos descubrimos algunas cuestiones relevantes con relación a la innovación y la creación de nuevos mercados, considerando las condiciones institucionales que reducen o favorecen la incertidumbre en los mercados, contribuyen a su organización, facilitan los intercambios o bloquean y condicionan su desarrollo.

\section{Bibliografía}

Chandler, A. D. (1977). The Visible Hand. The Managerial revolution in American Business. Cambridge: Harvard University Press.

Clarke, S. (1991). Marx, Marginalism and Modern Sociology. London: Macmillan.

Cooley, Ch. H. (1899). «Personal Competition», Economic Studies, American Economic Association, IV, no 2.

Cooley, Ch. H. (1956). «Social Organisation», en Cooley, Ch. H., The Two Major Works of Charles H. Cooley. Social Organisation and Human Nature and the Social Order. Glincoe, IL: The Free Press, págs. 3-425.

Cooley, Ch. H. (1966). Social Process. IL: Southern Illinois University Press.

Cooley, Ch. H. (1969a)+ «The Theory of Transportation», American Economic Association, no 9 (3) (mayo de 1894). Reunido en Cooley, Ch. H., Sociological Theory and Social Research. New York, Augustus M. Kelley Publishers, págs. $17-120$.

Cooley, Ch. H. (1969b). «The Development of Sociology at Michigan», en Cooley, Ch. H., Sociological Theory and Social Research. New York: Augustus M. Kelley, Publishers, págs. 3-16.

Doвbin, F. (2005). «Comparative and Historical Approaches to Economic Sociology», en Smelser, N. J. y Swedberg, R., The Handbook of Economic Sociology. Princeton: Princeton University Press.

Durkheim, É. (1966). Lecciones de sociología. Física de las costumbres y el derecho. Buenos Aires: Schapire.

Durkheim, É. (1974). Las reglas del método sociológico. Madrid: Morata.

Durkheim, É. (1982). La división del trabajo social. Madrid: Akal.

Giner, S. (2004). Teoría sociológica clásica. 2a edición actualizada. Barcelona: Ariel.

Gislain, J. J. y Steiner, P. (1995). La Sociologie économique 1890-1920. Paris: Presses Universitaires de France. 
Herranz, R. (2003). «Los fundamentos de la Nueva Sociología Económica y la Sociología de las Organizaciones», en PÁez, T., Sociología de las organizaciones. Caracas: Ceatpro.

Herranz, R. (2007). La sociología de los mercados internos de trabajo. Madrid: Consejo Económico y Social. Colección Estudios.

Herranz, R. (2008). "Georg Simmel y la Sociología Económica* el mercado, las formas sociales y el análisis estratégico», Papers, n. ${ }^{\circ}$ 87:269-286.

Macaulay, S. (1963). «Non Contractual Relations in Business», American Sociological Review, n. ${ }^{\circ}$ 28, 1, febrero, págs. 55-67.

Mowery, D. y Rosenberg, N. (1979). «The influence of markets demand upon innovation: a critical review of some recent empirical studies», Research Policy, n+ ${ }^{\circ}$, págs. 102-53.

Polanyi, K. (1989), La gran transformación. Madrid: La Piqueta.

Schmookler, J. (1966). Invention and Economic Growth. Cambridge, MA: Harvard University Press.

Schumpeter, J. A. (1976). Teoría del desenvolvimiento económico, México: Fondo de Cultura Económica.

Steiner, P.(2002). La Sociologie économique. Paris: La Decouverte.

Steiner, P. y Vatin, F. (2009). Traité de Sociologie Économique. Paris: Presses Universitaires de France.

Swedberg, R. (1991). Schumpeter. A Biography. Princeton: Princeton University Press.

Swedberg, R. (1994). «Markets as Social Structures», en Smelser, N. J. y Swedberg, R., The Handbook of Economic Sociology. Princeton: Princeton University Press.

Simmel, G. (1977). Filosofía del dinero. Madrid* Instituto de Estudios Políticos. Simmel, G. (1986)+ «Las grandes urbes y la vida del espíritu», en Simmel, G., El individuo y la libertad. Barcelona: Península.

Simmel, G. (1986a). Sociología. Estudios sobre las formas de socialización. Madrid: Alianza.

Simmel, G. (1988). Sobre la aventura. Ensayos filosóficos. Barcelona: Península. Tocqueville, A. de (1978). La democracia en América. Méxicoः FCE.

Weber, M. (1985). Economía y sociedad. Méxicoः FCE.

Weber, M. (1987). Historia económica general. México: FCE.

Weber, M. (1987a). La bolsa. Introducción al sistema bursátil. Barcelona: Ariel. Weber, Max (1994). Sociología del trabajo industrial. Madrid: Trotta. 